\title{
Accuracy of ultrasound to detect thrombosis in pregnancy: A systematic review
}

\author{
Aldemar Araujo Castro ${ }^{1}$, Fernando José Camello de lima², Célıo Fernando de Sousa-Rodrigues ${ }^{3}$, Fabiano Timbó Barbosa ${ }^{4 *}$ \\ ${ }^{1} \mathrm{MSc}$ in Vascular Surgery. Vascular Surgeon at Hospital Geral do Estado Professor Osvaldo Brandão Vilela, Maceió, AL, Brazil \\ ${ }^{2}$ PhD Student in Health Sciences. Assisting Professor of Human Anatomy, Universidade Federal de Alagoas (Ufal), Maceió, AL, Brazil \\ ${ }^{3} \mathrm{PhD}$ in Morphology. Adjunct Professor of Human Anatomy, Ufal and Universidade Estadual de Ciências da Saúde de Alagoas, Maceió, AL, Brazi \\ ${ }^{4} \mathrm{PhD}$ in Health Sciences. Adjunct Professor of Basics of Surgical and Anesthetic Technique, Ufal, Maceió, AL, Brazi
}

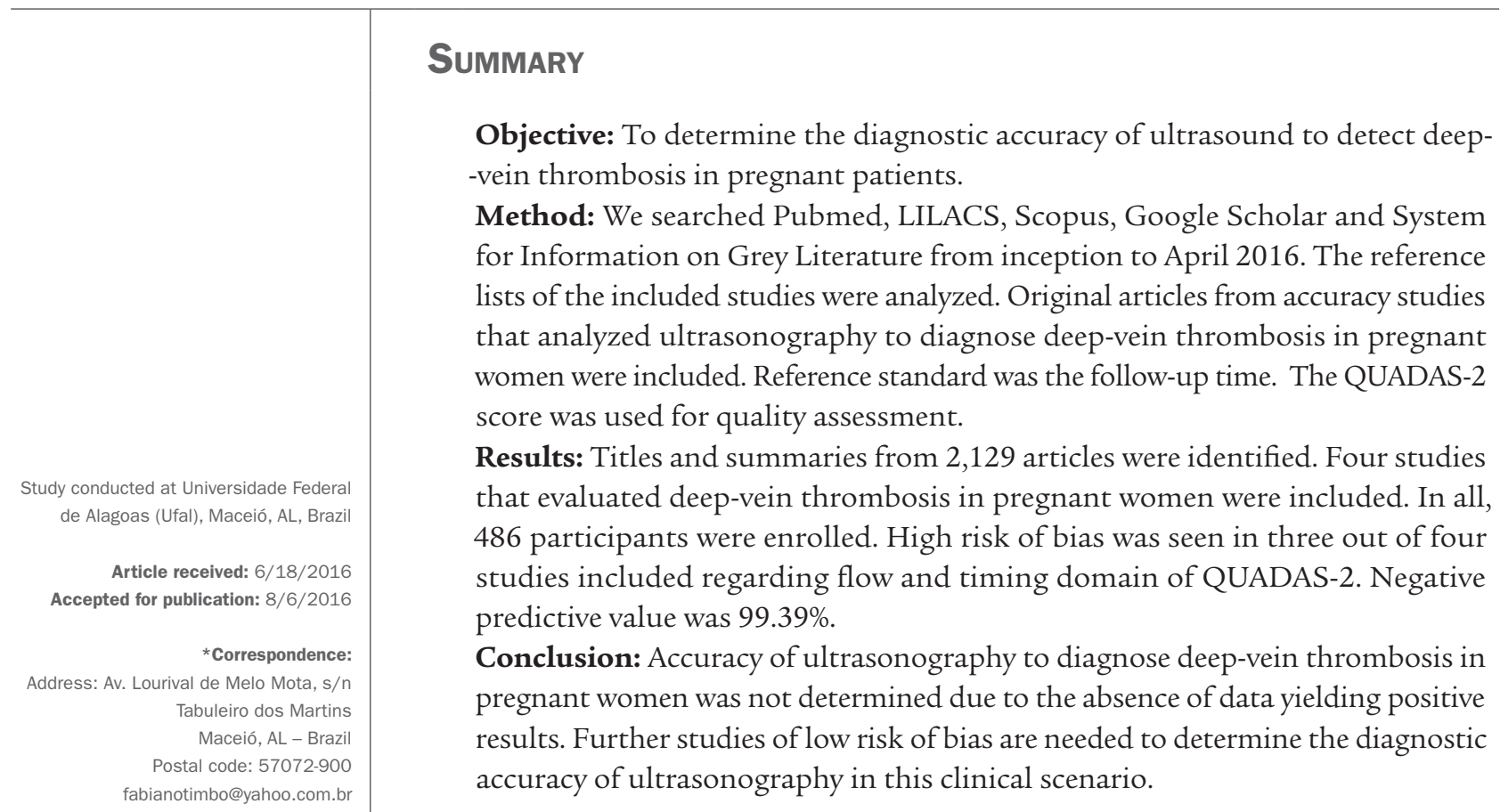

Keywords: pregnancy, ultrasonography, venous thrombosis.

\section{INTRODUCTION}

Pregnant women have an increased risk of thrombosis due to hypercoagulability state that protects against bleeding in childbirth. ${ }^{1}$ Pregnancy increases the risk of thrombosis three- to five-fold. ${ }^{1,2}$ The venous system is more involved and can be identified in $75 \%$ of the cases. ${ }^{2}$ Deep-vein thrombosis (DVT) is a life-threatening condition and is one of the main causes of death during pregnancy in developed countries. ${ }^{3}$ DVT is difficult to diagnose during pregnancy because its symptoms, including swelling of the legs, edema and pelvic or back pain, can be caused by physiologic changes. ${ }^{3,4}$

Normal pregnancy and early puerperium have high D-dimer levels and its measurement in suspected cases is not recommended. ${ }^{5} \mathrm{~A}$ negative $\mathrm{D}$-dimer result can be seen in cases of venous thromboembolism. ${ }^{6}$

Diagnostic possibilities are compression ultrasonography of the leg veins, echocardiography and lung ultrasound. ${ }^{7}$ Compression ultrasonography can be performed within two minutes. ${ }^{7}$ The sensitivity of clinical presentation combined with these diagnostic options is over $90 \%{ }^{7}$ The accuracy of ultrasonography is not determined when used alone, so the aim of our systematic review was to determine the diagnostic accuracy of ultrasound to detect deep-vein thrombosis in pregnant patients.

The hypothesis of this systematic review is that ultrasonography is an accurate diagnostic method to rule out deep-vein thrombosis in pregnant women.

\section{Method}

A protocol was developed a priori and is available from the corresponding author in case it needs to be analyzed. Institutions, journals or researchers did not influence our results. The Preferred Reporting Items for Systematic Reviews and Meta-Analyses (PRISMA) statement was followed to report this systematic review. ${ }^{8}$ This was a systematic review of diagnostic test accuracy studies. 


\section{Eligibility criteria}

- Types of study: Original articles from accuracy studies were included. Duplicated studies were not found in this review. Original articles with incomplete description of outcomes were excluded from this systematic review.

- Type of participants: Pregnant or postpartum women. We considered three months after delivery for inclusion in this systematic review.

- Index test: The index test was ultrasonography.

- Target condition: All participants have clinical suspicion of deep-vein thrombosis. D-dimer test could be used before ultrasonography.

- Reference standard: Our reference standard was follow-up time. We considered at least three months of follow-up time.

\section{Identification of studies}

The information was accessed from: Pubmed, Literatura Latino-Americana e do Caribe em Ciências da Saúde (LILACS), Scopus, Google Scholar, System for Information on Grey Literature (SIGLE). Each database was screened from inception to April 2016. Reference lists of the included original articles were also searched, but no new studies were identified. No restrictions were made regarding language, journal or document format. The search strategy used in Pubmed was adapted and used for the other databases.

The search strategies used in this systematic review were:

a. Pubmed: (("ultrasonography”[Subheading] OR "ultrasonography"[All Fields] OR "ultrasound"[All Fields] OR "ultrasonography”[MeSH Terms] OR "ultrasound"[All Fields] OR "ultrasonics"[MeSH Terms] OR "ultrasonics"[All Fields]) AND ("venous thrombosis"[MeSH Terms] OR ("venous"[All Fields] AND "thrombosis"[All Fields]) OR "venous thrombosis"[All Fields] OR ("deep"[All Fields] AND "vein"[All Fields] AND "thrombosis"[All Fields]) OR "deep vein thrombosis"[All Fields]) AND ("pregnancy”[MeSH Terms] OR "pregnancy”[All Fields])).

b. LILACS: ultrasound (thrombosis OR pregnant).

c. Scopus: ((TITLE-ABS-KEY (pregnancy)) AND (diagnostic ultrasound) AND (TITLE-ABS-KEY (thrombosis)).

d. Google Scholar: "diagnostic ultrasound" "venous thrombosis" OR "deep-vein thrombosis" pregnant OR pregnancy.

e. SIGLE: deep-vein thrombosis.

\section{Article selection}

Title, summaries or both, identified by the abovementioned search strategy for all databases were independently reviewed by two investigators. Studies on the diagnostic performance of ultrasound for DVT that were in accordance with our eligibility criteria were retrieved for reading of the full text. A standardized, pre-pilot format was developed by the authors and used to collect data. Any disagreements between investigators were resolved by consensus meetings.

\section{Quality assessment}

Quality assessment was made using Quality Assessment of Diagnostic Accuracy Studies (QUADAS-2). ${ }^{9}$ The QUADAS-2 tool has four domains: patient selection, index test, reference standard, and flow and timing. These domains were assessed in terms of risk of bias. ${ }^{9}$ The first three domains were assessed for applicability. Three responses were possible: low, high or unclear. Signaling questions from QUADAS-2 tool helped to appraise the risk of bias. ${ }^{9}$

\section{Outcomes}

The primary outcome was accuracy of ultrasound. Secondary outcomes included: positive predictive value, negative predictive value, sensibility and specificity of the ultrasound.

\section{Data analysis}

We summarized data from all included studies. We planned to extract true-positives, true-negatives, false-positives and false-negatives and enter the information in $2 \times 2$ tables. These data were used to calculate sensitivity, specificity, positive predictive value, negative predictive value and accuracy. Review Manager (RevMan) software version 5.1 (Cochrane Collaboration, Oxford, England) was used to analyze data.

\section{RESULTS}

\section{Study selection}

A flow diagram demonstrating the selection process is outlined in Figure 1. In all, 2,129 titles and summaries were screened and five original articles were selected for full-text analysis. One study was excluded, which is explained in Figure 1. Four original articles were included from quality assessment. ${ }^{10-13}$ We also analyzed 79 titles from the reference lists of the four studies above, but we did not find any additional article.

Four studies were identified according to the selection process. Although three included studies that analyzed only women with negative results, the authors concluded 
that ultrasonography is a safe method to rule out the diagnosis of DVT..$^{10,11,13}$ One study evaluated the agreement between ultrasonography and magnetic resonance imaging. ${ }^{12}$ This study was not included in quantitative analysis (Figure 1). ${ }^{12}$ In all, 486 participants were enrolled in these four studies. Characteristics of the studies included can be seen in details in Table 1 .

\section{Quality assessment}

The methodological assessment chart shows the percentage of low, high and unclear results for quality domain (Figure 2). The graph shows some potential areas of concern. Considering risk of bias, three out of four studies were classified as high risk of bias for the flow and timing domains due to participants being excluded from analysis. ${ }^{10,11,13}$ Other domains were classified as unclear or low. One study did not report if the sample was consecutive or random; ${ }^{10}$ three studies did not report if the reference standard results were interpreted without knowledge of the results of the index test. ${ }^{10,11,13}$ Considering applicability, one study reported the agreement between ultrasound and magnetic resonance imaging. ${ }^{12}$

\section{Outcomes}

Three of the studies could be used in the analysis..$^{10,11,13}$ They did not report true-positive cases and false-positive

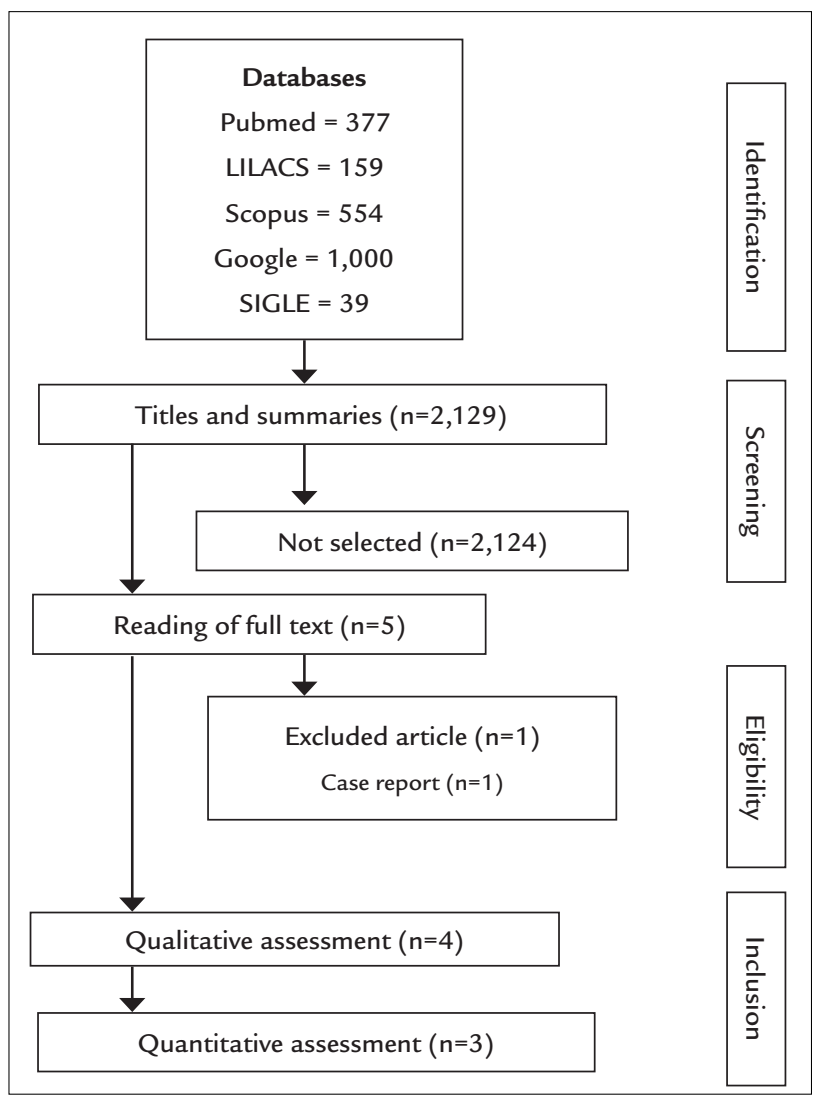

FIGURE 1 Flow diagram summarizing the selection process.

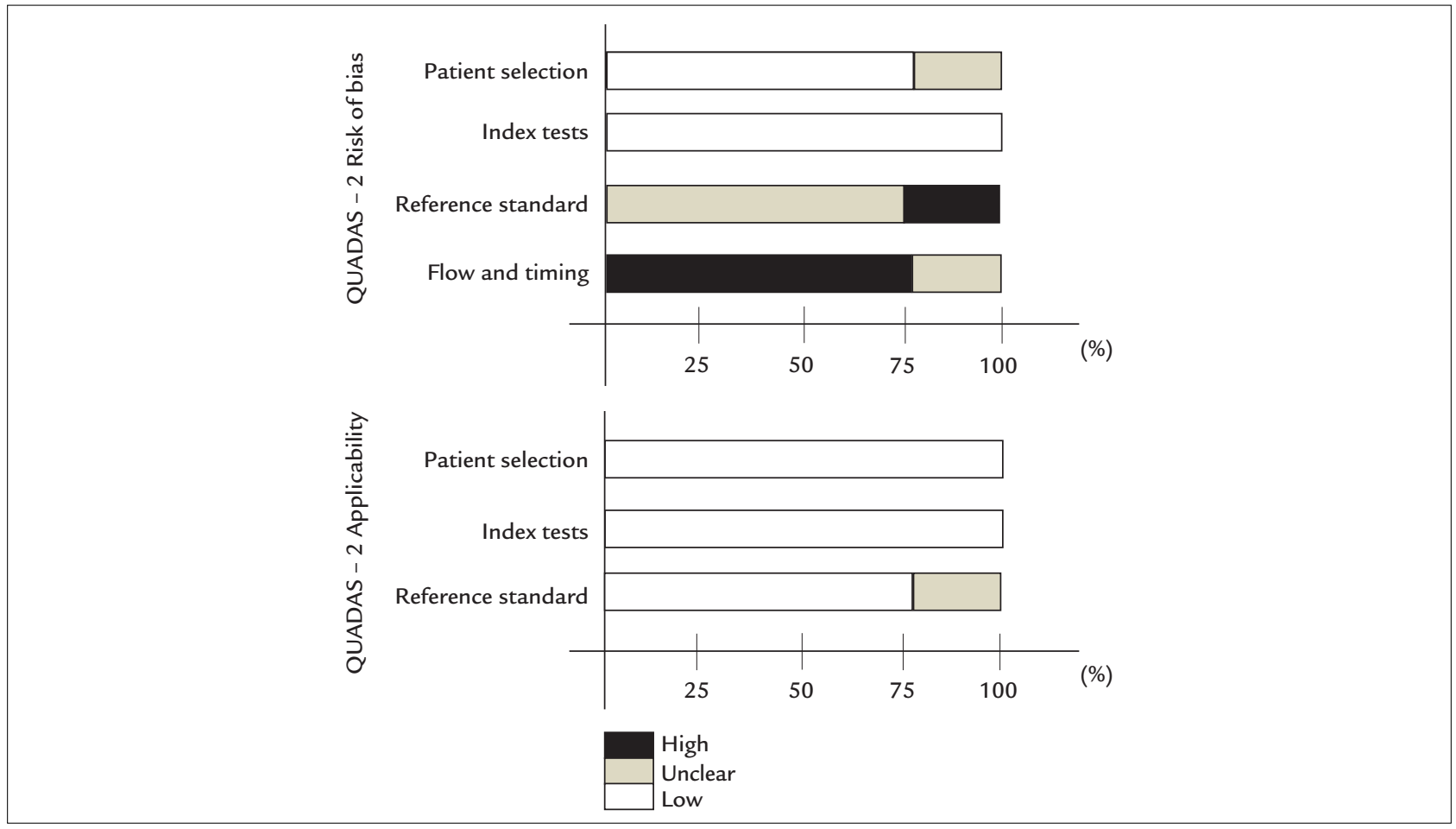

FIGURE 2 Graphical representation of quality assessment. 


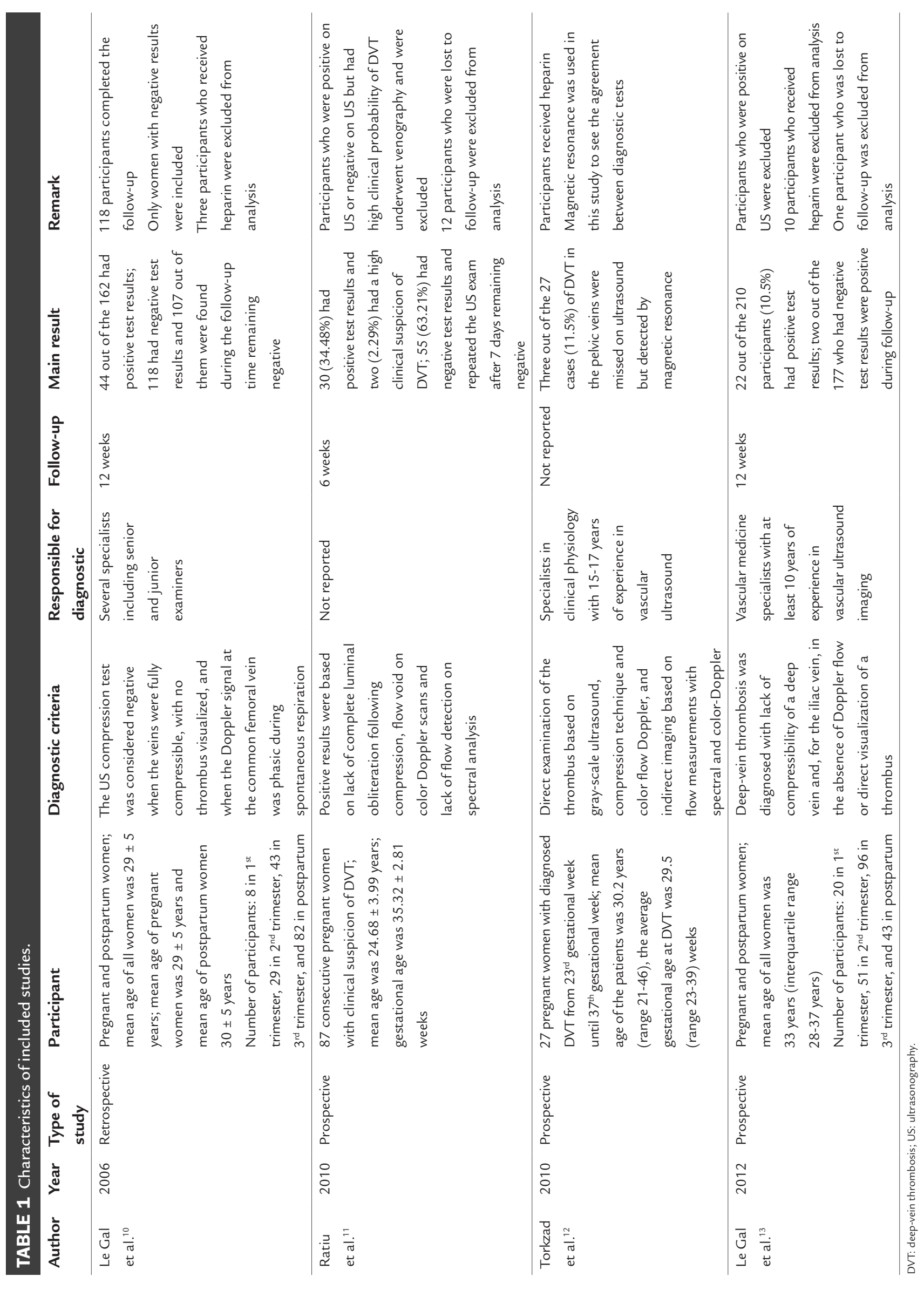


cases. ${ }^{10,11,13}$ Meta-analysis was not possible. We could not determine accuracy, sensibility, specificity and positive predictive value. Data from the three studies were used to calculate negative predictive value. ${ }^{10,11,13}$ The negative predictive value was $99.39 \%$.

\section{Discussion}

Our hypothesis was not confirmed in this systematic review. Although four accuracy studies were identified, in three of them only patients with negative results were analyzed, ${ }^{10,11,13}$ and in one of them there was an agreement between ultrasonography and magnetic resonance imaging. ${ }^{12}$ We determined predictive negative value that was $99.39 \%$.

Our main problem was analyzing the negative cases without positive cases. Positive cases received anticoagulant therapy and were excluded from the analysis. ${ }^{10,11,13}$ One systematic review published in 2006 that analyzed DVT and pulmonary embolism concluded that only two studies gave support to treat pregnant women but only if there was a high clinical suspicion and normal results from serial plethysmography. ${ }^{14}$ The absence of data on true-positive and false-positive cases resulted in 100\% specificity, but this value is not true. Accuracy, sensitivity and positive predict value could not be estimated in this systematic review.

The studies included analyzed pregnant and postpartum women with high suspicion of DVT. None of the studies included reported the prevalence of DVT. Blinding of outcome assessment was not reported and the authors reported more than one specialist analyzing the participants. We do not know if these specialists analyzed different participants alone or together. The authors did not report agreement between specialists. One author classified his study as prospective, but the follow-up time was not reported. ${ }^{12}$ In this study, more positive cases could be diagnosed if the time of the follow-up was described and adequate.

The QUADAS-2 is a tool for systematic review and helps to evaluate the quality of accuracy of studies. ${ }^{9}$ Two domains were problematic considering the risk of bias: reference standard and flow and timing. It was unclear if the results of the reference standard were interpreted without knowledge of the index test results. Blinding of this process is necessary to determine the best accuracy of the ultrasonography. Some participants could not be analyzed because they were lost during the follow-up. These participants could be positive cases and the outcomes of this systematic review could be misleading. We considered low concerns about applicability.
Negative predictive value totaled $100 \%$ in two of the studies ${ }^{10,11}$ and $98.87 \%$ in another one. ${ }^{13}$ Our result was $99.39 \%$. Systematic reviews and meta-analyses aim to summarize the accuracy of diagnostic tests. ${ }^{15}$ We cannot determine other parameters and the accuracy of ultrasonography for DVT in pregnant women is unknown.

In current clinical practice, pregnant women who have a positive diagnosis of DVT based on ultrasonography are treated with heparin, which is considered more conservative than other treatments. The studies included in our review analyzed lower-limbs of pregnant and postpartum women, yielding negative results based on ultrasonography alone, disregarding other tests such as D-dimer. No definite conclusion can be drawn from negative ultrasonography results. It is more conservative follow the women during pregnancy when a negative result and a high clinical suspicion are present.

Further studies of low risk of bias are needed to determine the diagnostic accuracy of ultrasound to detect deep-vein thrombosis in pregnant patients. In particular, authors have to analyze patients with positive results from ultrasonography with other diagnostic tests such as D-dimer, plethysmography and clinical presentation.

\section{Conclusion}

The accuracy of the ultrasonography to diagnose deep-vein thrombosis in pregnant women was not established due to lack of data from positive results. Negative predictive value was $99.39 \%$. Further studies of low risk of bias are needed to determine the diagnostic accuracy of ultrasonography in this scenario.

\section{Resumo}

Acurácia da ultrassonografia para detectar trombose na gravidez: uma revisão sistemática

Objetivo: Determinar a acurácia diagnóstica da ultrassonografia para detectar trombose venosa profunda (TVP) em pacientes grávidas.

Método: Pubmed, Lilacs, Scopus, Google Acadêmico e System for Information on Grey Literature foram pesquisados do início até abril de 2016. As referências dos estudos incluídos foram avaliadas. Artigos originais de estudos de acurácia que analisaram ultrassonografia para diagnosticar trombose venosa profunda em mulheres grávidas foram incluídos. O teste de referência foi o tempo de seguimento. O escore de QUADAS-2 foi usado para avaliar a qualidade. 
Resultados: Títulos e resumos de 2.129 artigos foram identificados. Quatro estudos que avaliaram trombose venosa profunda em grávidas foram incluídos. No total, 486 participantes foram selecionadas. Alto risco de viés foi visto em três dos quatro estudos incluídos considerando o domínio fluxo e tempo do QUADAS-2. O valor preditivo negativo foi $99,39 \%$.

Conclusão: A acurácia da ultrassonografia para diagnosticar trombose venosa profunda em mulheres grávidas não pôde ser determinada em razão da ausência de dados de resultados positivos. Estudos com baixo risco de viés são necessários para determinar a acurácia diagnóstica da ultrassonografia nesse cenário clínico.

Palavras-chave: gravidez, ultrassonografia, trombose venosa.

\section{References}

1. James AH. Pregnancy and thrombotic risk. Crit Care Med. 2010; 38(2 Suppl):S57-63.

2. James AH. Thrombosis in pregnancy and maternal outcomes. Birth Defects Res C Embryo Today. 2015; 105(3):159-66.

3. Simcox LE, Ormesher L, Tower C, Greer IA. Pulmonary thrombo-embolism in pregnancy: diagnosis and management. Breathe (Sheff). 2015; 11(4):282-9.

4. Chunilal SD, Bates SM. Venous thromboembolism in pregnancy: diagnosis, management and prevention. Thromb Haemost. 2009; 101(3)428-38.
5. Khalafallah AA, Morse M, Al-Barzan AM, Adams M, Dennis A, Bates G, et al. D-dimer levels at different stages of pregnancy in Australian women: a single centre study using two different immunoturbidimetric assays. Thromb Res. 2012; 130(3):e171-7.

6. To MS, Hunt BJ, Nelson-Piercy C. A negative D-dimer does not exclude venous thromboembolism (VTE) in pregnancy. J Obstet Gynaecol. 2008; 28(2):222-3.

7. Mathis G. [Ultrasound in thromboembolism]. Praxis (Bern 1994). 2015; 104(19):1013-8.

8. Moher D, Liberati A, Tetzlaff J, Altman DG; PRISMA Group. Preferred reporting items for systematic reviews and meta-analyses: the PRISMA statement. PLoS Med. 2009; 6(7):e1000097.

9. Whiting PF, Rutjes AW, Westwood ME, Mallett S, Deeks JJ, Reitsma JB, et al. QUADAS-2: a revised tool for the quality assessment of diagnostic accuracy studies. Ann Intern Med. 2011; 155(8):529-36.

10. Le Gal G, Prins AM, Righini M, Bohec C, Lacut K, Germain P, et al. Diagnostic value of a negative single complete compression ultrasound of the lower limbs to exclude the diagnosis of deep venous thrombosis in pregnant or postpartum women: a retrospective hospital-based study. Thromb Res. 2006; 118(6):691-7.

11. Ratiu A, Navolan D, Spătariu I, Biris M, Miculiță M, Motoc A. Diagnostic value of a negative single color duplex ultrasound in deep vein thrombosis suspicion during pregnancy. Rev Med Chir Soc Med Nat Iasi. 2010; 114(2):454-6.

12. Torkzad MR, Bremme K, Hellgren M, Eriksson MJ, Hagman A, Jörgensen T, et al. Magnetic resonance imaging and ultrasonography in diagnosis of pelvic vein thrombosis during pregnancy. Thromb Res. 2010; 126(2):107-12.

13. Le Gal G, Kercret G, Ben Yahmed K, Bressollette L, Robert-Ebadi H, Riberdy L, et al.; EDVIGE Study Group. Diagnostic value of single complete compression ultrasonography in pregnant and postpartum women with suspected deep vein thrombosis: prospective study. BMJ. 2012; 344:e2635.

14. Nijkeuter M, Ginsberg JS, Huisman MV. Diagnosis of deep vein thrombosis and pulmonary embolism in pregnancy: a systematic review. J Thromb Haemost. 2006; 4(3):496-500.

15. Leeflang MM. Systematic reviews and meta-analyses of diagnostic test accuracy. Clin Microbiol Infect. 2014; 20(2):105-13. 